\title{
MONITORING LACTIC ACID CONCENTRATIONS BY INFRARED SPECTROSCOPY: A NEW DEVELOPED METHOD FOR LACTOBACILLUS FERMENTING MEDIA WITH POTENTIAL FOOD APPLICATIONS
}

\author{
A. Păucean, D.C. Vodnar, V. Mureșan*, F. Fetea, F. Ranga, S.M. Man, S. Muste \\ and C. SOCACIU \\ Faculty of Food Science and Technology, University of Agricultural Sciences and Veterinary Medicine \\ of Cluj-Napoca, 3-5 Calea Mănăștur Street, 400372, Cluj-Napoca. Romania
}

(Received: 7 December 2016; accepted: 24 February 2017)

\begin{abstract}
The lactic acid bacteria are key microorganisms for the production and preservation of fermented dairy products, cheeses, sourdough bread, and lacto-fermented vegetables. This study was developed to monitor lactic acid produced by Lactobacillus plantarum ATCC 8014 and Lactobacillus casei ATCC 393, as single strains and combined, in fermenting media by Fourier Transform Infrared Spectroscopy coupled to multivariate statistical analysis. Media containing different mixtures of carbohydrates were chosen as model fermenting media for monitoring lactic acid concentration by infrared spectroscopy, due to the fact that vegetable and animal food matrices could contain different carbohydrates as carbon sources. Three different types of media were obtained by adding different carbohydrates to a basic MRS medium. HPLC was used as reference method for lactic acid quantification. The calibration set $(n=36)$ was used for building model, while a validation set $(n=13)$ for testing the robustness of the developed model. The coefficients of determination between predicted and reference values were 0.986 and 0.965 , while root mean square error for calibration and validation sets recorded values of 0.127 and $0.263 \mathrm{~g} \cdot \mathrm{l}^{-1}$, respectively. Results confirmed the efficiency of FTIR spectroscopy combined with multivariate statistics, as a rapid, reliable, and cost-effective tool for routine monitoring of lactic acid.
\end{abstract}

Keywords: chemometric methods, FTIR, lactic acid, Lactobacillus

Lactic acid bacteria (LAB) are used for production and preservation of fermented dairy products, cheeses, sourdough bread, and also lacto-fermented vegetables (ZALAN et al., 2010), where they contribute to important qualities such as taste, texture, and shelf life (HoRn et al., 2005). In the present study, a FTIR method for determination of lactic acid production in fermenting media by Lactobacillus plantarum ATCC 8014 and Lactobacillus casei ATCC 393 was investigated. The two types of LAB were selected due to their high adaptability to different substrates and growing conditions. Lactobacillus plantarum is a facultative heterofermentative strain, widely used in a variety of food fermentations, with good adaptation ability due to its high metabolic flexibility to challenging environments. Lactobacillus casei is also adaptable to diverse conditions, being capable of metabolizing a variety of carbohydrates and to shift homo/heterolactic fermentation profiles.

\footnotetext{
* To whom correspondence should be addressed.

Phone: +40 264596 384; fax: +40 264593 792; e-mail: vlad.muresan@usamvcluj.ro
}

This is an open-access article distributed under the terms of the Creative Commons Attribution 4.0 License, which permits unrestricted use, distribution, and reproduction in any medium for non-commercial purposes, provided the original author and source are credited. 
High-performance liquid chromatography (HPLC) has been the commonly accepted technique used in organic acid determination (Sivakesava et al., 2001; Moreira \& Santos, 2005; Liu et al., 2011). However, liquid chromatography is time consuming and requires sample handling (centrifugation, filtration, dilution, etc.). Therefore, the HPLC-based technique is considered to be an invasive and indirect analytical method. Fourier-transform infrared (FTIR) analysis coupled with data-processing algorithms has become a powerful tool (MoReira \& SANTOS, 2005). In FTIR spectroscopy, results can be processed (among other methods) by partial least squares (PLS) regression, an extension of the multiple linear regression model (VANDEN BRANDEN \& HUBERT, 2004).

Investigating the scientific literature related to the analysis of chemical compounds from different systems (culture media, food, blood, plasma, etc.) by means of infrared spectroscopic methods, it was revealed that in the last decade, there is an increasing trend for developing new methods. So far as we know, there have not yet been any studies on the infrared spectroscopic measurement of lactic acid by means of FTIR spectroscopy and chemometrics, directly on a fermenting media containing Lactobacillus plantarum ATCC 8014 and Lactobacillus casei ATCC 393. Several studies have focused on near infrared control during the L-(+)-lactic acid production (GONZÁLEZ-VARA et al., 2000), monitoring of fermentation processes for Lactobacillus fermentum ES15 (Tosi et al., 2003), or FT-MIR and FT-Raman spectroscopic techniques in Lactobacillus casei fermentation from media containing only glucose at varying concentrations as carbon sources (SivakeSAVA et al., 2001). Quantitative infrared spectroscopy has also been used for monitoring glucose and gluconic acids (FrANCO et al., 2006) or for lactic acid production from whey (TRIPATHI et al., 2015), for organic acids determination in fruit vinegars (LiU et al., 2011), wine (REGMI et al., 2012; MARTELO-VIDAL \& VÁzquez, 2014a), fruit (Bureau et al., 2009), and tomatoes (BEullens et al., 2009), as well as for detection of polyphenolic compounds from red wine (MARTELo-VIDAL \& VÁzQUEZ, 2014b) and nutritional compounds determination from yogurt (Moros et al., 2006) and cheese (Subramanian et al., 2011). Most of these quantitative determinations were validated by multivariate statistics or chemometrics. Thus, the main objective of our study was to develop a rapid and simple FTIR method and a calibration model to monitor lactic acid concentrations during lactic acid fermentation by Lactobacillus plantarum ATCC 8014 and Lactobacillus casei ATCC 393 in model fermentation media containing different carbohydrates.

\section{Materials and methods}

\subsection{Microorganisms and media}

L. plantarum ATCC 8014 and L. casei ATCC 393 were purchased in lyophilized form (Microbiologics, Minnesota, USA) and grown in de Man, Rogosa, Sharpe (MRS) broth (Merck, Darmstadt, Germany). Inoculum cultures were obtained as was described by PAUCEAN and co-workers (2013). All microorganisms were grown until the late exponential phase (ca. $10^{9} \mathrm{CFU} \cdot \mathrm{ml}^{-1}$, colony forming units per $\mathrm{ml}$ ) was achieved. Actively growing cultures of $L$. plantarum and $L$. casei, separately or in a mixture of $1: 1$, were transferred into media using an inoculation rate of $1.5 \%$ and a mean cell density of $10^{8} \mathrm{CFU} \cdot \mathrm{ml}^{-1}$. A basic, non-commercial MRS medium (containing $15 \mathrm{~g} \cdot 1^{-1}$ yeast extract, $8 \mathrm{~g} \cdot \mathrm{l}^{-1}$ beef extract, $10 \mathrm{~g} \cdot \mathrm{l}^{-1}$ peptone, $\left.2 \mathrm{~g} \cdot \mathrm{l}^{-1} \mathrm{~K}_{2} \mathrm{HPO}_{4}, 0.1 \mathrm{~g} \cdot \mathrm{l}^{-1} \mathrm{MgSO}_{4} * 7 \mathrm{H}_{2} \mathrm{O}, 0.05 \mathrm{~g} \cdot \mathrm{l}^{-1} \mathrm{MnSO}_{4} * \mathrm{H}_{2} \mathrm{O}\right)$ with the initial $\mathrm{pH}$ adjusted to 6.2 prior sterilization $\left(121^{\circ} \mathrm{C}, 20 \mathrm{~min}\right)$ was prepared from separate components and then was supplemented with carbohydrates mixtures. Three types of carbohydrate 
mixtures were added: glucose $0.2 \%$ and sucrose $0.2 \%$ (D), glucose $0.2 \%$ and fructose $0.2 \%$ (B), glucose $0.2 \%$ and maltose $1.5 \%$ (C). The name of the samples contain as first letter the media type (D, B, or C), followed by microorganism codification ( $\mathrm{p}$ for L. plantarum or $\mathrm{c}$ for $L$. casei), and sampling time $(4,8,24)$. For example, the sample Dcp8 was the sample containing $0.2 \%$ glucose and $0.2 \%$ sucrose, inoculated with both LAB strains (L. plantarum and L. casei) taken after $8 \mathrm{~h}$ of fermentation.

\subsection{High performance liquid chromatography (HPLC)}

The high-performance liquid chromatography with UV detection (HPLC-UV) (Agilent Technologies 1200 Series) coupled with an HPLC column Acclaim OA $5 \mu \mathrm{m}, 4 \times 250 \mathrm{~mm}$ was applied (Paucean et al., 2013).

\subsection{Fourier transform infrared spectroscopy (FTIR)}

As an alternative to HPLC, FTIR spectroscopy was used to identify the specific marker in fermentation processes, namely lactic acid. The FTIR absorbance spectra $\left(650-4000 \mathrm{~cm}^{-1}\right)$ were recorded using a FTIR spectrometer (IR-Prestige, Shimadzu Europa GmbH, Duisburg, Germany) equipped with a horizontal attenuated total reflectance (ATR) accessory with single reflection. The spectral resolution was $4 \mathrm{~cm}^{-1}$ and 128 scans were accumulated for each spectrum.

\subsection{Spectral data analysis}

For lactic acid prediction, the PLS regression from the Unscramble 10.1, Camo ASA Norway, was used. The specific region used for data analysis was $600-1800 \mathrm{~cm}^{-1}$. Preliminary trials revealed that the utilization of the first-derivative transformed FTIR spectra was optimal by comparing the regression results in terms of coefficients of determination $\left(\mathrm{R}^{2}\right)$ and rootmean-square errors. The first-derivative FTIR spectra were calculated based on the SavitzkyGolay procedure, averaging left side and right side four points and using a second degree polynomial. The FTIR and HPLC correlation was represented by R-square. Model assessment and predictive capability was evaluated by coefficient of determination $\left(\mathrm{R}^{2}\right)$, root mean square error of calibration (RMSEC) or prediction (RMSEP). Robust models must have coefficients of determination close to unit and low RMSEC and RMSEP values.

\section{Results and discussion}

\subsection{Monitoring lactic acid content in fermenting media by HPLC}

The reference data of lactic acid content during lactic fermentation in media were obtained by HPLC technique (Paramithiotis et al., 2007; Vodnar et al., 2010; Tripathi et al., 2015). Lactic acid was eluted at $t_{R}=3.1 \mathrm{~min}$. The calibration curve of lactic acid standard was linear $\left(\mathrm{R}^{2}=0.9989\right)$ when the concentration ranged between 8 and $8000 \mathrm{mg} \cdot \mathrm{l}^{-1}$ with a least-square regression line $\mathrm{Y}=4.86 \mathrm{X}+563.15$ (where, $\mathrm{Y}$ is the area of lactic acid peak and $\mathrm{X}$ is the injected volume in $\mathrm{mg} \cdot \mathrm{l}^{-1}$ ). This indicated that the chemical values of lactic acid obtained by these linear regression functions were accurate and correct. Values of lactic acid $\left(\mathrm{mg} \cdot \mathrm{l}^{-1}\right)$ produced in culture media with different content and type of carbohydrates ranged between $1446 \mathrm{mg} \cdot 1^{-1}$ 
and $5889 \mathrm{mg} \cdot \mathrm{l}^{-1}$ during the $24 \mathrm{~h}$ of fermentation. Generally, the lowest content of lactic acid was produced after $4 \mathrm{~h}$, while the highest content was reached after $24 \mathrm{~h}$ of fermentation under the conditions of this study. Briefly, in the case of L. plantarum, the smallest value was $1508 \mathrm{mg} \cdot \mathrm{l}^{-1}$, while the highest value recorded was $4794 \mathrm{mg} \cdot \mathrm{l}^{-1}$. For $L$. casei, the smallest and the highest contents of lactic acid were $252.3 \mathrm{mg} \cdot \mathrm{l}^{-1}$ and $4489 \mathrm{mg} \cdot \mathrm{l}^{-1}$, respectively. When the same media were inoculated with both microorganisms, L. plantarum and L. casei, the smallest content of lactic acid was $1750.1 \mathrm{mg} \cdot \mathrm{l}^{-1}$, while the highest value recorded was 5889 $\mathrm{mg} \cdot \mathrm{l}^{-1}$. The obtained results were consistent with previous researches (PARAMithiotis et al., 2007; VRANCKEN et al., 2008) suggesting that lactic acid production during lactic acid fermentation was strain dependent. Moreover, it was significantly influenced by the available carbohydrates.

\subsection{Spectra description}

Figure 1 shows the spectrum of standard lactic acid (Fig. 1A) and the spectra for the analysed samples after $4 \mathrm{~h}$ of fermentation (Fig. 1B). The samples were obtained by inoculating different media with single strains of lactobacilli, L. plantarum, L. casei, or a co-culture of these two strains. The applicable wavelength was selected by preparing 3 replicates of the same sample containing lactic acid. All spectra were characterized by common absorption bands. Spectra of media showed absorbance bands at $927 \mathrm{~cm}^{-1}, 1051 \mathrm{~cm}^{-1}, 1730 \mathrm{~cm}^{-1}$, $2939 \mathrm{~cm}^{-1}$, and $2989 \mathrm{~cm}^{-1}$. These bands were representative of the chemical groups of components present in the media. Assignment of functional groups corresponding to the vibration modes was based on identification of the spectrum peaks and matching the frequency with the chemical groups that absorb in the IR region (Sivakesava et al., 2001). The $\mathrm{C}=\mathrm{O}$ stretching band at $1730 \mathrm{~cm}^{-1}$ is characteristic of carboxylic acid groups. The peaks ranging between $2500-3000 \mathrm{~cm}^{-1}$ are due to the $\mathrm{O}-\mathrm{H}$ stretching of the acid component, while the bands in the region $1200-950 \mathrm{~cm}^{-1}$ are explained by stretching modes of $\mathrm{C}-\mathrm{C}$ and $\mathrm{C}-\mathrm{O}$ functional groups. The lower range of the region below $1200 \mathrm{~cm}^{-1}$ generally represents different kinds of $\mathrm{C}-\mathrm{H}, \mathrm{C}-\mathrm{O}$, and $\mathrm{CH}_{3}$ vibrations (rocking, deformation, stretching) (BUREAU et al., 2009; Vodnar et al., 2010; Regmi et al., 2012; Tripathi et al., 2015). Furthermore, multivariate data analysis (below section) corroborated the importance of $600-1800 \mathrm{~cm}^{-1}$ region for lactic acid FTIR spectroscopic characterization, this region showing the highest coefficients of determination.

As compared to standard lactic spectrum, the spectra obtained by FTIR showed a noisy region between 1300 and $1800 \mathrm{~cm}^{-1}$, probably caused by bacterial cells, as the media samples were analysed without any prior filtrations. These conditions were selected as the development of a new, rapid, cost-effective method for monitoring lactic acid in fermenting media requiring no sample preparation was intended. However, this inconvenience is usually solved by data pre-treatments (Savitzky Golay procedure) as will be further demonstrated in chemometric techniques section.

\subsection{Monitoring lactic acid concentrations by infrared spectroscopy coupled to chemometrics}

All samples analysed by the reference HPLC method were tested by FTIR to obtain the infrared spectra. PLS regressions were subsequently developed. Several calibration models for predicting lactic acid content were built using PLS regression by optimizing the spectral region and pre-treatments. The selection criteria for best PLS model were the coefficients of 
A

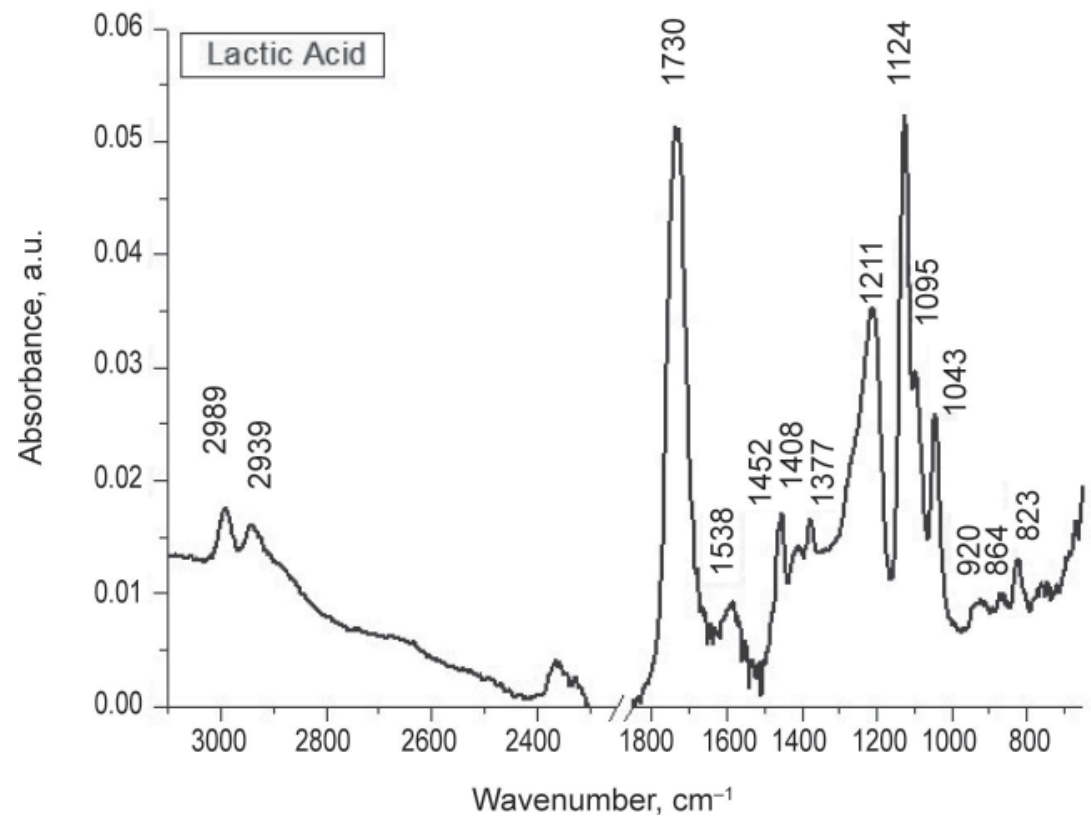

B

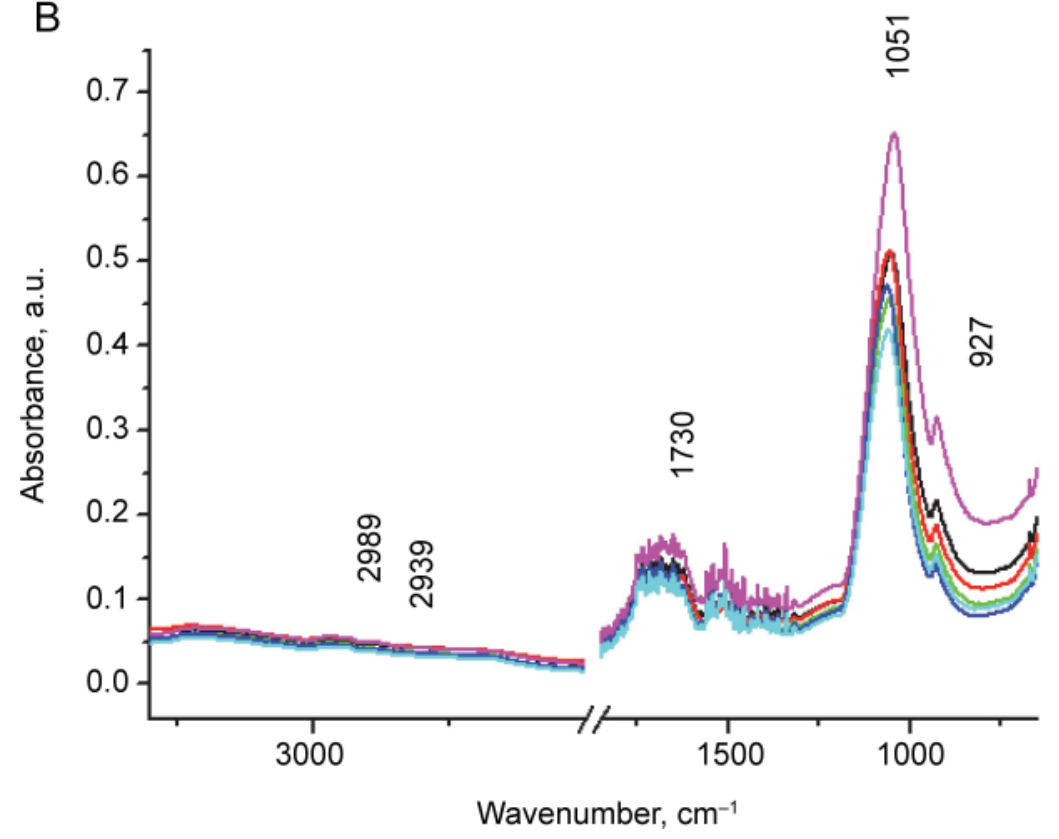

Fig. 1. FTIR spectrum for lactic acid standard (A) and FTIR spectra for media (B)

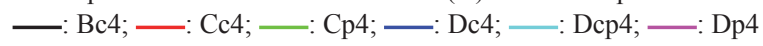


determination, RMSEC, and RMSEP, while the slope and offset were taken into consideration for distinguishing systematic errors and studying the correlation between the reference and FTIR models (Fig. 2). The calibration set $(n=36)$ was used for building the model, while the validation set $(n=13)$ was used for testing the robustness of the developed model. The prediction results for calibration and validation sets generated by the best PLS model are shown in Table 1. The PLS calibration method gave encouraging results for monitoring lactic acid content in culture media. The optimum number of factor was 4 . The coefficients of determination $\left(\mathrm{R}^{2}\right)$ between the predicted and the reference values were 0.986 and 0.965 , while RMSEC and RMSEP for calibration and validation sets recorded values of 0.1268 and $0.2632 \mathrm{~g} \cdot \mathrm{l}^{-1}$, respectively. The calibration method gave satisfactory results for estimation of lactic acid concentration. The coefficient of determination very close to unity and the considerably lower RMSEC value indicated that the data were well modelled using the lower wave number spectral range with 1st derivative pre-processing approach. Validation results show that the FTIR spectroscopic method could accurately predict the concentration of lactic acid produced by lactobacilli in fermenting media, as demonstrated by the obtained values for $\mathrm{R}^{2}$ and RMSEP.

Table 1. The prediction results of lactic acid content in validation set by PLS models (for details see Materials and methods sections)

\begin{tabular}{llll}
\hline Sample & \multicolumn{2}{c}{ Lactic acid $\left(\mathrm{mg} \cdot \mathrm{l}^{-1}\right)$} & Error of prediction, \\
$\%$ & Predicted & 0.98 \\
\cline { 2 - 4 } Dc 24 & Reference & 4115.6 & 1.02 \\
Dc 8 & 4202.4 & 3689.5 & 1.11 \\
Dcp 8 & 3629.7 & 3084.1 & 0.93 \\
Dp 4 & 2785.0 & 1748.2 & 0.99 \\
Dp 8 & 1884.7 & 4764.0 & 0.93 \\
Bc 24 & 4793.7 & 2392.4 & 1.06 \\
Bc 4 & 2572.9 & 1613.5 & 0.95 \\
Bp 24 & 1518.0 & 1429.4 & 1.05 \\
Cc 4 & 1507.7 & 1513.5 & 0.99 \\
Cc 8 & 1445.9 & 2150.8 & 1.05 \\
Ccp 8 & 2162.9 & 3085.9 & 0.98 \\
Cp 24 & 2929.2 & 4118.6 & 0.98 \\
Cp 4 & 4198.3 & 3296.5 & - \\
\hline $\mathbf{R}^{2}$ & 3371.7 & 0.965 & - \\
\hline F & 0.986 & & \\
\hline
\end{tabular}

$\mathrm{R}^{2}$ : The coefficient of determination; F: Optimal number of factors 


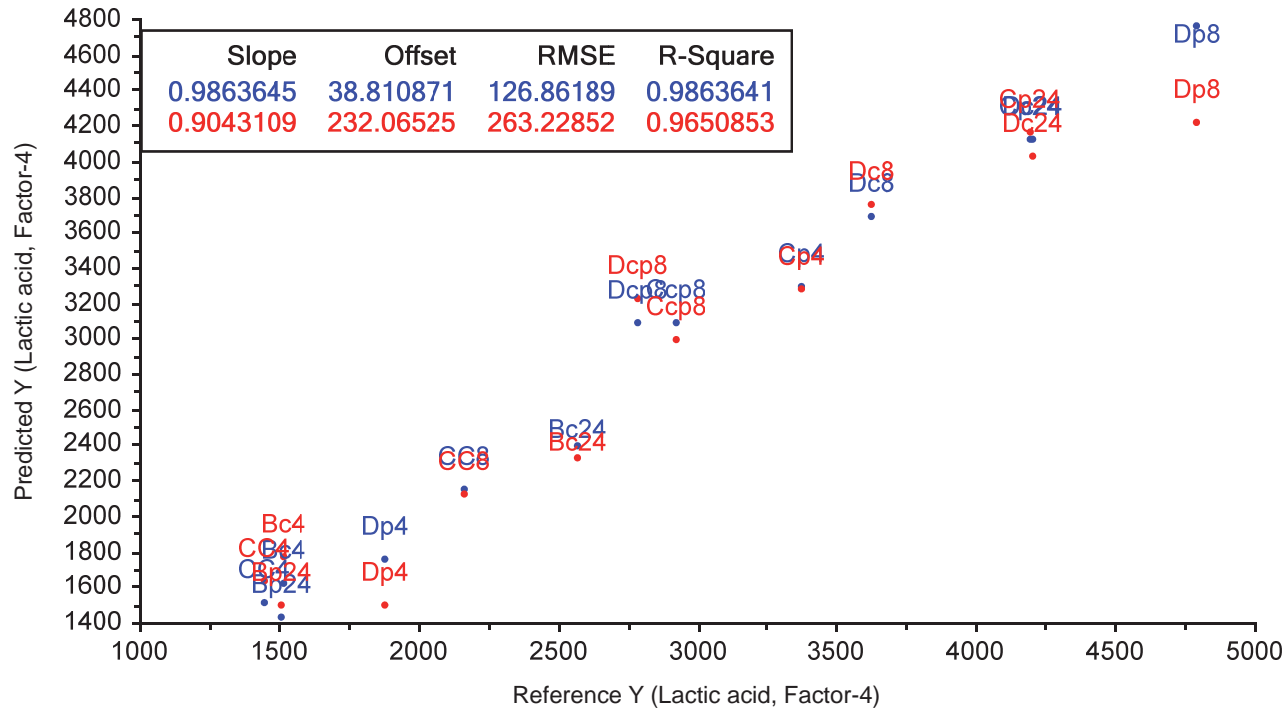

Fig. 2. Linear regression calibration curve for optimized PLS calibration model

\section{Conclusions}

This study demonstrated the potential of FTIR spectroscopy coupled with multivariate analysis methods as a tool to monitor lactic acid concentrations in fermenting media. This approach will provide a suitable methodology for monitoring the kinetics of the whole process in real time. Future investigations will be conducted in order to extend FTIR procedure utilization to monitor the dynamics of the lactic acid fermentation in sourdough systems and bread-making technology.

This work was supported by a grant of the Romanian National Authority for Scientific Research and Innovation, CNCS/CCCDI-UEFISCDI, project number PN-III-P2-2.1-BG-2016-0122 within PNCDI III.

\section{References}

Beullens, K., Kirsanov, D., Irudayaraj, J., Rudnitskaya, A., Legin, A., Nicolaï, B.M. \& Lammertyn, J. (2006): The electronic tongue and ATR-FTIR for rapid detection of sugars and acids in tomatoes. Sensor. Actuat. B-Chem., 116, 107-115.

Bureau, S., Ruiz, D., Reich, M., Gouble, B., Bertrand, D., Audergon, J.M. \& Renard, C.M. (2009): Application of ATR-FTIR for a rapid and simultaneous determination of sugars and organic acids in apricot fruit. Food Chem., 115, 1133-1140.

Franco, V.G., Perín, J.C., Mantovania, V.E. \& Goicoechea, H.C. (2006): Monitoring substrate and products in a bioprocess with FTIR spectroscopy coupled to artificial neural networks enhanced with a genetic-algorithmbased method for wavelength selection. Talanta, 68(3), 1005-1012.

González-Vara, Y.R.A., Vaccari, G., Dosi E, Trilli, A., Rossi, M. \& Matteuzzi, D. (2000): Enhanced production of L-(+)-lactic acid in chemostat by Lactobacillus casei DSM 20011 using ion-exchange resins and cross-flow filtration in a fully automated pilot plant controlled via NIR. Biotechnol. Bioeng., 67(2), 147-156. 
Horn, S.J., Aspmo, S.I. \& Eusink, V.G.H. (2005): Growth of Lactobacillus plantarum in media containing hydrolysates of fish viscera. J. Appl. Microbiol., 99, 1082-1089.

Liu, F., HE, Y., WANG, L. \& Sun, G. (2011): Detection of organic acids and pH of fruit vinegars using near-infrared spectroscopy and multivariate calibration. Food Bioprocess Tech., 4, 1331-1340.

Martelo-Vidal, M.J. \& Vázquez, M. (2014a): Evaluation of ultraviolet, visible, and near infrared spectroscopy for the analysis of wine compounds. Czech J. Food Sci., 32, 37-47.

Martelo-Vidal, M.J. \& VÁzquez, M. (2014b): Determination of polyphenolic compounds of red wines by UVVIS-NIR spectroscopy and chemometrics tools. Food Chem., 158(1), 28-34.

Moreira, H.L. \& SANTOS, L. (2005): Analysis of organic acids in wines by fourier-transform infrared spectroscopy. Anal. Bioanal. Chem., 382, 421-425.

Moros, J., Iñón, F.A., Khanmohammadi, M., Garrigues, S. \& de la Guardia, M. (2006): Evaluation of the application of attenuated total reflectance-Fourier transform infrared spectrometry (ATR-FTIR) and chemometrics to the determination of nutritional parameters of yogurt samples. Anal. Bioanal. Chem., 385,708-715.

Paramithiotis, S., Sofou, A., Tsakalidou, E. \& Kalantzopoulos, G. (2007): Flour carbohydrate catabolism and metabolite production by sourdough lactic acid bacteria. World J. Microbiol. Biot., 23, 1417-1423.

Paucean, A., Vodnar, D.C., Socaci, S.A. \& Socaciu, C. (2013): Carbohydrate metabolic conversions to lactic acid and volatile derivatives, as influenced by Lactobacillus plantarum ATCC 8014 and Lactobacillus casei ATCC 393 efficiency during in vitro and sourdough fermentation. Eur. Food Res. Technol., 237, 679-689.

Regmi, U., Palma, M. \& Barroso, C.G. (2012): Direct determination of organic acids in wine and wine-derived products by Fourier transform infrared (FT-IR) spectroscopy and chemometric techniques. Anal. Chim. Acta, 732, 137-144.

Sivakesava, S., Irudayaraj, J. \& Ali, D. (2001): Simultaneous determination of multiple components in lactic acid fermentation using FT-MIR, NIR, and FT-Raman spectroscopic techniques. Process Biochem., 37, 371-378.

Subramanian, A., Alvarez, V.B., Harper, W.J. \& Rodriguez- Saona, L.E. (2011): Monitoring amino acids, organic acids, and ripening changes in Cheddar cheese using Fourier-transform infrared spectroscopy. Int. Dairy J., $21,434-440$.

Tosi, S., Rossi, M., Tamburini ,E., Vaccari, G., Amaretti, A. \& Matteuzzi, D. (2003): Assessment of in-line nearinfrared spectroscopy for continuous monitoring of fermentation processes. Biotechnol. Progr., 19, 1816-1821.

Tripathi, A.D., Srivastava, S.K., Singh, P., Singh, R.P., Singh, S.P., Jha, A. \& Yadav, P. (2015): Optimization of process variables for enhanced lactic acid production utilizing paneer whey as substrate in SMF. Appl. Food Biotechnol., 2(2), 46-55.

VAnden Branden, K. \& Hubert, M. (2004): Robustness properties of a robust PLS regression method. Anal. Chim. Acta, 515, 229-241.

Vodnar, D.C., Paucean, A., Dulf, F.V. \& Socaciu, C. (2010): HPLC characterization of lactic acid formation and FTIR fingerprint of probiotic bacteria during fermentation processes. Not. Bot. Horti Agrobo., 38(1), 109-113.

Vrancken, G., Rimaux, T., De Vuyst, L. \& Leroy, F. (2008): Kinetic analysis of growth and sugar consumption by Lactobacillus fermentum IMDO 130101 reveals adaptation to the acidic sourdough ecosystem. Int. J. Food Microbiol., 128, 58-66.

Zalan, Z., Hudacek, J., Stetina, J., Chumchalova, J. \& Halasz, A. (2010): Production of organic acids by Lactobacillus strains in three different media. Eur. Food Res. Technol., 230, 395-404. 